\title{
Applying a time-limit model of movement frequency to field data: is it possible?
}

\author{
Emmanuelle Turpin-Legendre, Jean-Pierre Meyer \\ INRS (National Institute for Safety and Research), Vandoeuvre les Nancy, France \\ Emmanuelle.turpin-legendre@inrs.fr
}

Keywords: Repetitive gesture; Musculoskeletal disorders; Standardization

\section{Introduction}

One way to prevent musculoskeletal disorders (MSD) of the upper limbs during a repetitive gesture is to reduce the frequency of the movements [1]. Although the choice of the European standardization group is to limit the frequencies of the actions of a repetitive task, few objective data allow for the definition of such limits (NF EN 1005-5, 2007). A limit curve of local strain of a muscle or muscle group for repetitive contractions does not really exist.

The objective of this work was to develop in the laboratory and validate in the field a model that links time-limit to frequency of movement during repetitive tasks. Keeping work below the limit of exhaustion should reduce MSD.

\section{Method}

Three experiments (Figure 1) allowed the construction of a model that links time-limit to frequency of movement during simple repetitive bending-extension gestures of the forearm or arm for experiments 1 and 2 and a complex gesture more representative of real work activities for the third experiment. A total of 60 young healthy subjects participated in 3 successive experiments. In the 3 experiments, combinations of load ( 0 to $3 \mathrm{~kg}$ ) and frequency ( 50 to $85 \%$ of maximal frequency) were tested. Endurance time-limit (Elim) was the duration recorded until the subjects stopped the task due to exhaustion. Frequency of movement is expressed in percent of the maximal frequency ( $\%$ Fmax) obtained for all the loads.

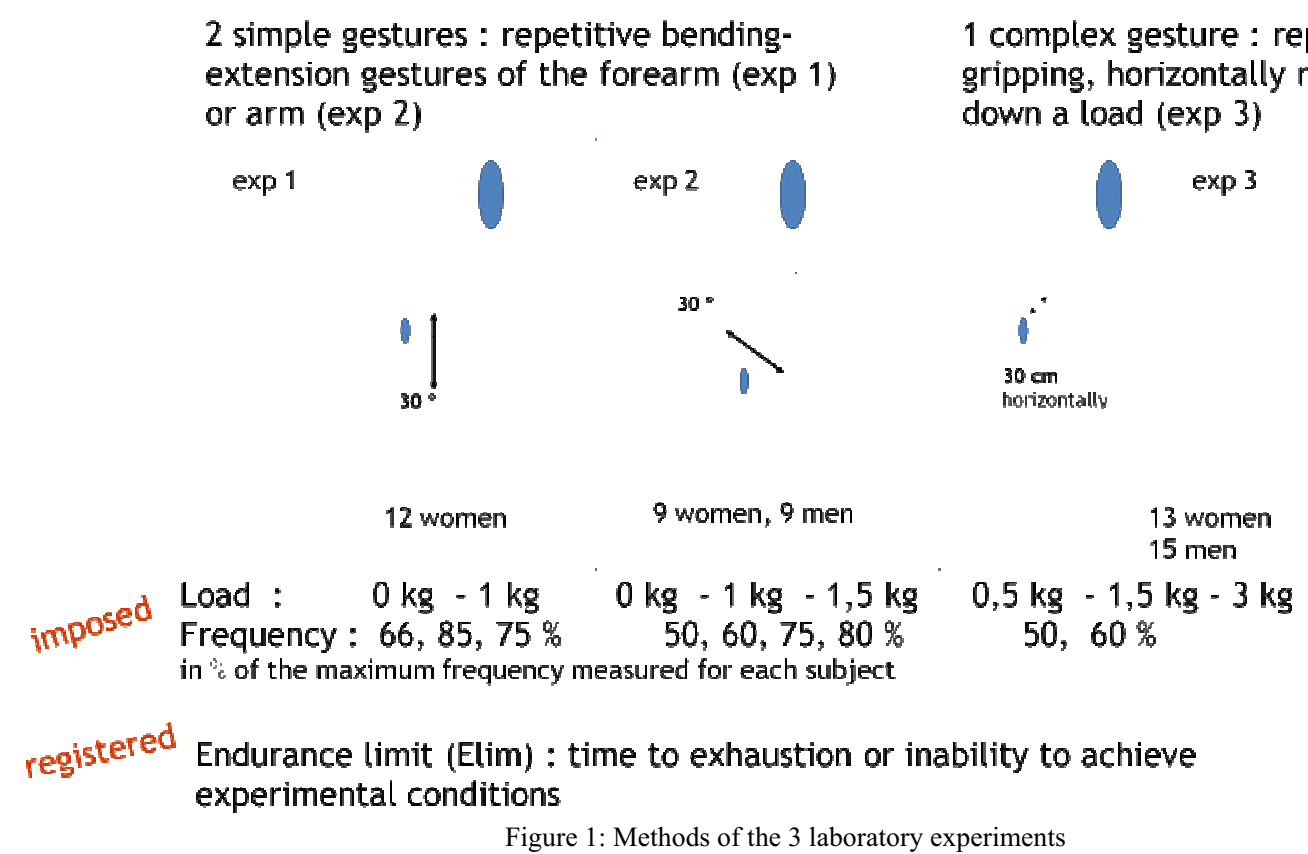

1051-9815/12/\$27.50 @ 2012 - IOS Press and the authors. All rights reserved 
Practical applications in the field were carried out to validate the model. Six factories where handling low loads at high frequency is common have participated. These factories were in the perfume, cutting meat, car assembly, wood furniture, car equipments and metalwork sectors. The repetitive task studied had to include a few gestures and short cycle times. Eighty employees were questioned about their health, the difficulties of the task and the perceived fatigue. The duration, movement frequency and maximal movement frequency defined for each task and each employee were recorded. Subjective evaluations and heart rate were used to assess the relative load of the task.

\section{Results-Discussion}

Figure 2 shows the time-limit curve as a function of movement frequency expressed in percent of the maximal frequency. The field data were added.

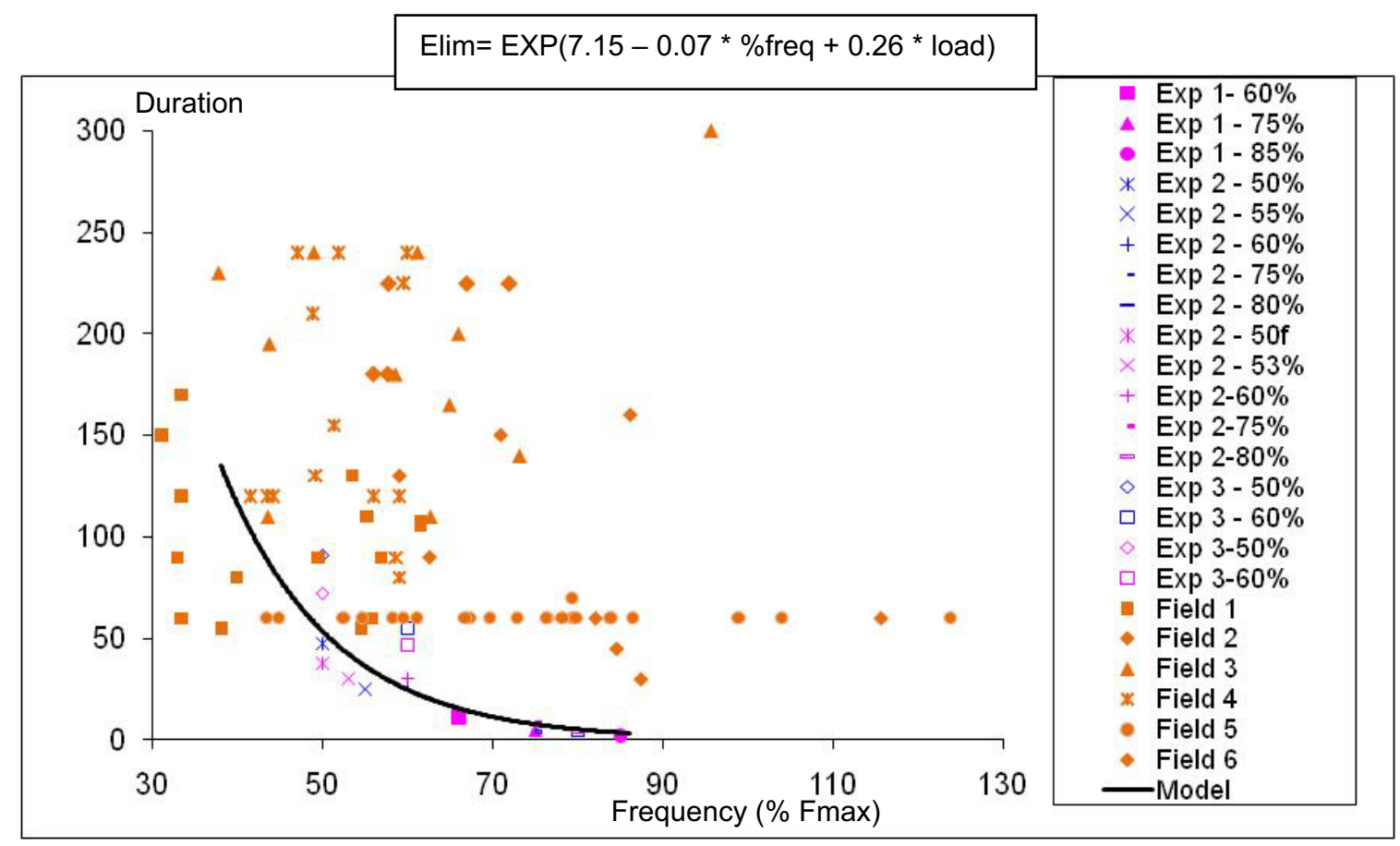

Figure 2: Model of duration (endurance limit in laboratory experiments) as a function of frequency of movement in percent of the maximal frequency (\% Fmax) and the field data (durations of the task as a function of frequency).

The field data are above the experimental limit curve but subjective data and heart rate show the not exhausting work of the employees. In the field, the employees had great difficulty in carrying out their habitual gesture at a maximum speed, which explains the extreme points in the frequency of gestures in Figure 2. Frequency of movement expressed in percent of the maximal frequency cannot be applied in the field.

Field data could be presented by the number of technical actions (TA) per minute with the OCRA method [2], like in Standard NF EN 1005-5 [3], but it is impossible to use the OCRA index which gives zero TA in the first two laboratory experiments. Number of gestures per minute could be use with field and laboratory data but the relation time-limit with number of gestures per minute is not significant in laboratory.

\section{Conclusion}

It is difficult to find the optimal parameter applicable in field and in laboratory studies.

The time-limit model is interesting for physiological research but it is not possible to apply it in the field. Maybe the tasks performed in laboratories are not complex enough, include too few gestures per minute, or are too far from reality?

\section{References}

[1] Byström, S.E.G., Kilbom, A. (1990). Physiological response in the forearm during and after isometric intermittent handgrip, Eur. J. Appl. Physiol., 60, 457-466.

[2] Colombini, D., Occhipinti, E., Grieco, A., (2002). Risk assessment and management of repetitive movements and exertions of upper limbs: Job analysis, OCRA risk index, prevention strategies and design principles. Elsevier Ergonomics; vol.2.

[3] NF EN 1005-5 (2007) - Safety of machinery - Human physical performance - Part 5 : Risk assessment for repetitive handling at high frequency. AFNOR. 